\title{
Article \\ Elastic and Inelastic Cross Sections for Low-Energy Electron Collisions with CIF Molecule Using the R-Matrix Method
}

\author{
Monika Bassi ${ }^{1}$, Anand Bharadvaja ${ }^{2, *(1)}$ and Kasturi Lal Baluja ${ }^{3}$ \\ 1 Department of Physics, Kalindi College, University of Delhi, New Delhi 110008, India; \\ monikabassi@kalindi.du.ac.in \\ 2 Department of Physics, Bhaskaracharya College of Applied Sciences, University of Delhi, \\ New Delhi 110075, India \\ 3 Department of Physics and Astrophysics, University of Delhi, New Delhi 110007, India; kl_baluja@yahoo.com \\ * Correspondence: anand_bharadvaja@yahoo.com
}

Citation: Bassi, M.; Bharadvaja, A.; Baluja, K.L. Elastic and Inelastic Cross Sections for Low-Energy Electron Collisions with ClF Molecule Using the R-Matrix Method. Atoms 2022, 10, 8. https://doi.org/10.3390/ atoms 10010008

Academic Editors: Dhanoj Gupta, Suvam Singh and Paresh Modak

Received: 26 December 2021

Accepted: 17 January 2022

Published: 19 January 2022

Publisher's Note: MDPI stays neutral with regard to jurisdictional claims in published maps and institutional affiliations.

Copyright: (c) 2022 by the authors. Licensee MDPI, Basel, Switzerland. This article is an open access article distributed under the terms and conditions of the Creative Commons Attribution (CC BY) license (https:// creativecommons.org/licenses/by/ $4.0 /)$.

\begin{abstract}
The CIF molecule belongs to an interhalogen family and is important in laser physics and condensed phase molecular dynamics. The elastic and excitation scattering cross sections are obtained in a fixed nuclei approximation using the UKRmol+ codes based on $R$-matrix formalism. The scattering calculations were performed in the static-exchange (SE), static-exchange-plus-polarisation (SEP), and close-coupling (CC) models. Three CC models with different target states were employed, namely, the 1-state, 5-states, and 12-states. In the CC model, the target states were represented by configuration interaction (CI) wavefunctions. A good agreement of dipole and quadrupole moments of the ground state was obtained with the experimental values, which indicates a good representation of the target modelling. The study predicted the existence of a shape resonance in the SE, SEP, and 5-states CC models. This resonance vanished in the 12-states CC model. The excitation cross sections from ground to the lowest two excited states were also reported. The elastic differential and momentum transfer cross sections were obtained in the 12-states CC models. The contribution of long-range interactions to elastic scattering was included via Born closure approach. The quantities like collision frequencies and rate coefficients were also presented over a wide range of electron temperatures. The ionization cross sections were computed using the binary-encounter-Bethe (BEB) model. The results were reported in $C_{2 v}$ point group representation.
\end{abstract}

Keywords: electron collision; scattering cross sections; ionization; excitation; $R$-matrix method; $\mathrm{BEB}$ model; resonances; plasma; CC model

\section{Introduction}

Chlorine monofluoride $(\mathrm{ClF})$ is the first (lightest) of six diatomic interhalogens. The isolated molecules of $\mathrm{CIF}$ in rare-gas matrices provide an opportunity to study molecular dynamics and chemistry in condensed phases both experimentally and theoretically. The interpretation of the photoelectron spectra of core- and valence-energy regions of this molecule is extremely useful in elucidating molecular electronic structure [1]. Several species from $\operatorname{ClF}_{n}(n=1-7)$ series are reactive in nature, which make them corrosive and oxidizing agents and are used in reactions with both organic and inorganic compounds [2]. The molecule is relevant as a laser source [3-5] and for laser-irradiation studies [6]. Its moderate etch rate [7] makes it suitable for a shallow etching even at high temperatures [8]. The measurements of electronic transitions of this molecule by UV femtosecond lasers have made possible the time-resolved measurements of the dynamical processes [9]. The spectroscopic studies of ClF in cryogenic solutions are useful in molecular dynamics [10]. The flash photolysis-initiated $C l F_{x}(\mathrm{x}=1,3,5)$ chemical lasers produce vibrational-rotation laser emission [11,12]. Further, the ClF systems were also identified to produce pure rotational laser emission [13] and display high gain and extended durations, which make them potential chemical lasers [14]. 
There exist plenty of work on the spectroscopy of ClF molecule. The ground state of $\mathrm{ClF}$ is ${ }^{1} \Sigma^{+}$and is formed by coupling of the electrons in the singly occupied $3 p_{z}$ orbitals of $\mathrm{Cl}$ and $2 p_{z}$ of $\mathrm{F}$ orbitals. The excited state of ${ }^{3} \Pi$ is formed via recoupled pair bonding when the $\mathrm{Cl}$ atom is rotated in such a way that one of its $3 p^{2}$ orbital gets aligned along the internuclear axis. The longer bond of the triplet state in comparison to its singlet state results in smaller bond energy. Alekseyev et al. had computed spectroscopic constants by accounting for the relativistic effects [15]. Li et al. employed configuration interactions and coupled-cluster methods in theory structure calculations [16]. Both these works are considered to be very accurate. There also exist several structure studies for $\mathrm{ClF}$ and $\mathrm{ClF}^{-}$ involving different quantum mechanical methods [2,17-21].

The low-energy interactions of electrons with molecules are of fundamental interest and play a significant role in many modern-day technological applications such as lasers [22], ignition of internal combustion engines [23], and low-temperature plasma applications $[24,25]$. The electron collisions with molecules initiate the plasma-etching processes upon which semiconductor industries rely. Thus, it is essential to know the electron collision cross sections to model a relevant phenomenon. The $R$-matrix method studies are available only for the electron attachment process to the interhalogen compounds [26]. These calculations revealed that the $C l F^{-}$electronic state is bound for $R=3.05 \mathrm{a}_{0}$, while for other values of internuclear distance the electronic state of this anion is a resonant state. However, there is no comprehensive study of the electron impact on this molecule. In the present work, we addressed this issue by employing the ab-initio R-matrix formalism [27] to calculate various collisional parameters.

\section{R-Matrix Method}

The $R$-matrix theory involves the division of configuration space into two regions: an inner and an outer. These regions are separated by a spherical boundary of radius $a$. In the inner region, the Hamiltonian eigenvalue problem was solved for $N+1$ electrons. The trial $N+1$ scattering eigenfunction in a closed coupling expansion scheme is expressed as [28]:

$$
\psi_{k}^{N+1}=A \sum_{i} \psi_{i}^{N}\left(x_{1}, \ldots, x_{N}\right) \sum_{j} \zeta_{j}\left(x_{N+1}\right) a_{i j k}+\sum_{m} \chi_{m}\left(x_{1}, \ldots, x_{N+1}\right) b_{m k}
$$

where $A$ is the anti-symmetrization operator, $\psi_{i}^{N}$ is the wavefunction of the $i$ th target state, and $a_{i j k}$ and $b_{m k}$ are the variational coefficients. The $x_{N}$ is the space and spin coordinate of the $N$ th target electron, and $\zeta_{j}$ is the continuum orbital spin-coupled with the scattering electron. The variational coefficients are determined by diagonalising the Hamiltonian matrix. The $\chi_{m}$ are the $L^{2}$ configurations and account for the dominant short-range correlations and polarisation effects in the inner region. These are the multi-center-quadratically integrable functions and are constructed by placing all the $N+1$ electrons in the target molecular orbitals (MOs). This method provides a good description of electron correlations when several excited states of the molecule are included. The static-exchange (SE) model includes only the ground Hartree-Fock (HF) state. The second sum in the SE approximation runs over the minimum number of configurations to relax the orthogonality constraints between target molecular orbitals and the functions used to represent the configuration. The scattering electron in the outer region is distinguishable from the target electrons, and the dominant short-range forces are negligible as the scattering electron is at a large distance from the centre of mass of the target. The electron propagates in the multipolar potentials of the molecule. A single-centre expansion of the wavefunction was carried out. The resulting set of coupled differential equations were solved by propagating the $R$-matrix computed at the spherical boundary to a large radial distance so that non-Coulombic potentials become negligible. This was done by using the Program RPROP [29]. These solutions were then matched with the asymptotic boundary conditions to extract the K-matrix from where scattering parameters like eigenphase sum and cross sections are determined. Generally, the $R$-matrix radius was taken as $12 \mathrm{a}_{0}$, and the propagation was carried out up to $100 \mathrm{a}_{0}$. 


\section{Target Description and Computational Details}

The $\mathrm{ClF}$ molecule has a natural point group of $C_{\infty v}$. The UK polyatomic $R$-matrix code UKRmol+ [30] version 3.0 [31] works using Abelian point groups up to $D_{2 h}$. The target and scattering calculations were, therefore, performed in $C_{2 v}$ molecular point group symmetry at the experimental equilibrium geometry $\mathrm{r}_{e}=1.618 \AA$ [32] using the $6-311 \mathrm{G}^{*}$ basis set for the $\mathrm{Cl}$ and $\mathrm{F}$ atoms. The self-consistent field (SCF) calculations yielded the ground $=$ state electronic configuration in $C_{2 v}$ point group as: $1 \mathrm{a}_{1}^{2}, 2 \mathrm{a}_{1}^{2} \ldots, 7 \mathrm{a}_{1}^{2}, 3 \mathrm{~b}_{1}^{2}, 3 \mathrm{~b}_{2}^{2}\left(\mathrm{X}^{1} A_{1}\right)$, which in its natural point group corresponds to $1 \sigma^{2}, 2 \sigma^{2} \ldots 7 \sigma^{2}, 1 \pi^{4} \ldots 3 \pi^{4}\left(\mathrm{X}^{1} \Sigma^{+}\right)$. The electronic target states were constructed using the occupied and virtual molecular orbitals obtained from HF-SCF calculations.

It is noticed from Tables 1 and 2 that the ground-state target properties like dipole moment, quadrupole moment $Q_{20}$, and vertical excitation energies of the target obtained at the $\mathrm{CI}$ level are in satisfactory agreement with the corresponding experimental values. The ionization energy of ClF from Koopman's theorem came out to $13.44 \mathrm{eV}$ and is close to the experimental value of $12.77 \mathrm{eV}$ [32]. The continuum basis set of Faure et al. [33] was employed to model the scattering electron. The continuum orbitals up to g partial waves i.e., $(l=4)$ were included. These were orthogonalised to the target molecular orbitals using Schmidt and Löwdin symmetric orthogonalisation methods. The polar molecules are known to suffer from slow convergence of the continuum orbital partial wave expansion [34]. To overcome this problem, the higher partial waves $1>4$ were accounted for via the Born Top-Up Program [22]. The calculations were performed in fixed-nuclei approximation by taking the $R$-matrix sphere radius of $12 \mathrm{a}_{0}$. At this radius, the cross sections were stable and the amplitude of occupied molecular orbitals was negligible $\left(\sim 10^{-6}\right)$ [35]. In the complete active space configuration interaction (CASCI) considered, 16 electrons were frozen in molecular orbitals $1-6 a_{1}{ }^{2}, 1 b_{1}^{2}, 1 b_{2}^{2}$, and 10 electrons were free to move in the orbitals $7-9 a_{1}^{2}, 2-4 b_{1}^{2}, 2-4 b_{2}^{2}$. This generated 1386 configuration state functions (CSFs) for $X^{1} A_{1}$ state, 1902 each for ${ }^{3} B_{1}$ and ${ }^{3} B_{2} ; 1302$ for ${ }^{1} B_{1}$ and ${ }^{1} B_{2} ;$ and 1854 for ${ }^{3} A_{1}$ states in the 12 -states CI model. Typically, the CSF for ${ }^{2} A_{1}$ scattering symmetry were 27,238 , and the number of scattering channels were 74 .

Table 1. Ground-state target properties for HF and 12-states CI model.

\begin{tabular}{cccc}
\hline Model & $\begin{array}{c}\text { Energy } \\
(\mathbf{a u})\end{array}$ & $\begin{array}{c}\text { Dipole Moment } \\
(\mathbf{a u})\end{array}$ & $\begin{array}{c}\text { Quadrupole Moment } \\
\left(\mathbf{Q}_{\mathbf{2 0}}\right)(\mathbf{a u})\end{array}$ \\
\hline HF & -558.8667 & 0.5545 & 0.9398 \\
12 -states CI & -558.9011 & $0.4363(0.353 *[32])$ & $1.0045(1.0 *[36])$ \\
\hline${ }^{*}$ : Experimental value. & &
\end{tabular}

The scattering models used in the present study are discussed briefly. In the SE model, only the static and exchange potentials are included. This is a unique model that can be easily checked by any other scattering formalism. It depends only upon the geometry of the target and the basis set employed. In the $R$-matrix method, the target polarisation is included via the inclusion of $L^{2}$ configurations. In the $n$-state CC models (where $\mathrm{n}=1,5$, and 12), we include the configuration interaction wavefunctions. In the scattering region, one electron from the HF configuration was promoted to a chosen subset of virtual orbitals, which in the present work consists of $\left(8 a_{1}, 9 a_{1}, 4 b_{1}, 4 b_{2}\right)$. This results in elastic and electronic excitation cross sections. In the SEP model, target polarisation was induced by promoting one occupied electron in the HF configuration to a set of virtual orbitals selected. 
Table 2. Vertical Excitation Energies (in eV) of the excited states for the target states in $C_{\infty v}$ and $C_{2 v}$ symmetries.

\begin{tabular}{lcc}
\hline Target State & Present Work & Other Works \\
\hline $1^{3} \Pi\left({ }^{3} B_{1},{ }^{3} B_{2}\right)$ & 3.84 & $\begin{array}{c}2.33 \text { (Expt) [37] } \\
3.10 \text { (CASSCF) [38] }\end{array}$ \\
\hline $1^{1} \Pi\left({ }^{1} B_{1},{ }^{1} B_{2}\right)$ & 5.10 & $\begin{array}{c}4.63 \text { (MRD-CI) [39] } \\
4.34 \text { (CI) [39] }\end{array}$ \\
\hline $2^{3} \Pi\left({ }^{3} B_{1},{ }^{3} B_{2}\right)$ & 8.33 & - \\
\hline $1^{3} \Sigma^{+}\left({ }^{3} A_{1}\right)$ & 8.64 & - \\
\hline $1^{3} \Delta_{2}\left({ }^{3} A_{2}\right)$ & 8.80 & - \\
\hline $2^{1} \Pi\left({ }^{1} B_{1},{ }^{1} B_{2}\right)$ & 9.01 & $\begin{array}{c}9.08 \text { (MRD-CI) [39] } \\
8.19 \text { (CI) [39] }\end{array}$ \\
\hline $1^{1} \Delta_{2}\left({ }^{1} A_{2}\right)$ & 9.45 & $\begin{array}{c}10.54 \text { (MRD-CI) [39] } \\
10.27 \text { (CI) [39] }\end{array}$ \\
\hline
\end{tabular}

In Figure 1a,b, we plotted the eigenphase sum for different scattering models employed corresponding to ${ }^{2} A_{1}$ symmetry only to detect any possible resonances. A resonance occurs when the eigenphase sum suddenly jumps by $\pi$ radians in a small energy range. The resonance parameters like $E^{r e s}$ and half-width $\Gamma^{r e s}$ can be extracted by fitting the eigenphase sums $\eta(E)$ to the Breit-Wigner profile [40].

$$
\eta(E)=\tan ^{-1}\left[\frac{\Gamma^{r e s}}{E-E^{r e s}}\right]+a_{1} E+a_{2} E^{2}
$$

In Equation (2), $a_{1}$ and $a_{2}$ are the fitting parameters, and $E$ is the projectile energy. The last two terms in the above equation define the quadratic energy background.
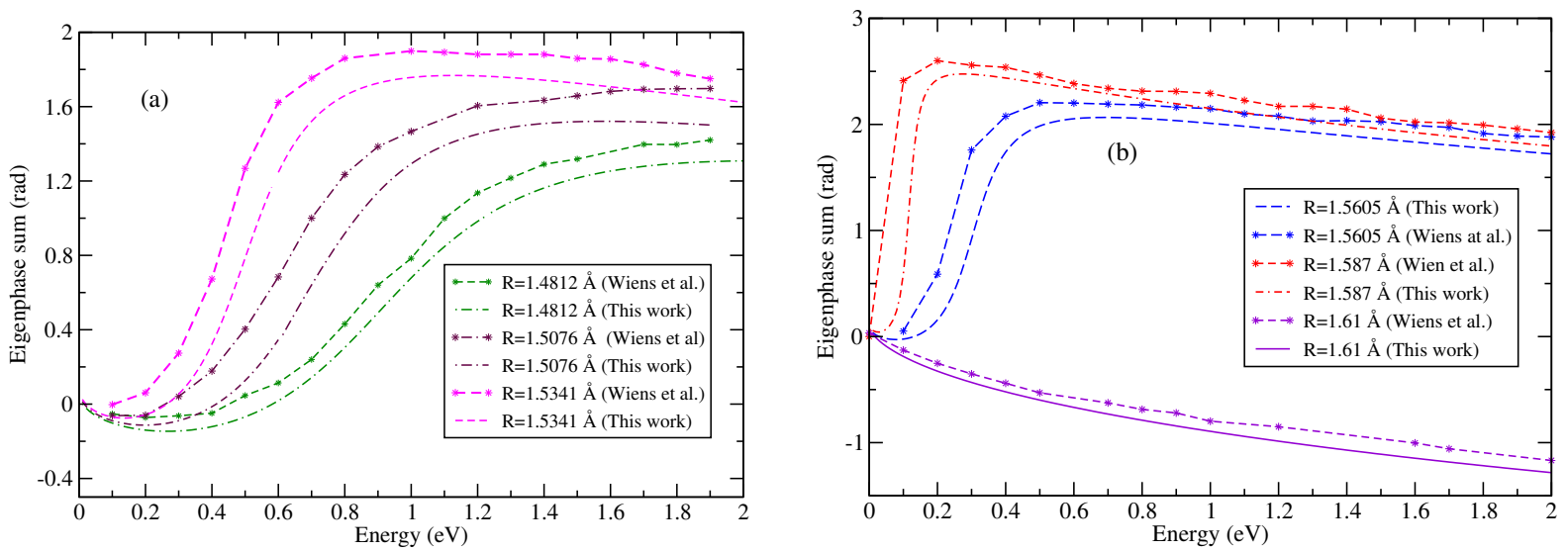

Figure 1. Eigenphase sum for the scattering symmetry ${ }^{2} A_{1}$ as a function of bond length in 12states CC model (a) $1.4812 \AA$ A: dotted dashed curve; this work, dotted dashed curve with star, Wiens et al. [26], $1.5076 \AA$ A: dashed dotted curve; this work, dashed dotted curve with star; Wiens et al., 1.5341 Å: dashed curve; this work, dashed curve with star, Wiens et al. (b) $1.5605 \AA$ : dashed curve; this work, dashed curve with star, Wiens et al., $1.587 \AA$ A: dashed dotted curve; this work, dashed dotted curve with star; Wiens et al., 1.61 A: line curve; this work, line curve with star, Wiens et al.

The symmetries like ${ }^{2} B_{1},{ }^{2} B_{2}$ and ${ }^{2} A_{2}$ do not exhibit any shape resonances and hence are not shown.

In Figure 2, we show resonance parameters as a function of the bond length in the 12-states CC model. The resonance values decrease with the increase in bond length. This shape resonance vanishes giving rise to a bound anionic state $\mathrm{ClF}^{-}$. From the first $\mathrm{R}$ matrix pole of ${ }^{2} A_{1}$ scattering symmetry that has the value of -558.9059 au and the energy 
$-558.9011 \mathrm{au}$ of the ground state, we obtain a value of $0.43 \mathrm{eV}$ for the vertical electron affinity (VEA), which is in excellent agreement with $0.44 \mathrm{eV}$ obtained using much larger basis sets [16,17]. The DFT value was $0.52 \mathrm{eV}$ [19].

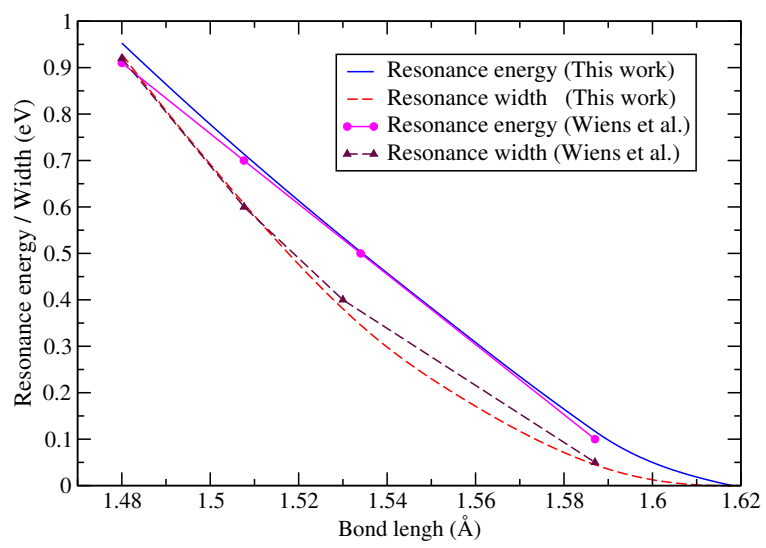

Figure 2. Resonance position and width for 12-states CC model: Resonance position-line curve; this work, line curve with circles; Wiens et al. [26]. Resonance width-dashed curve; this work, dashed curve with triangles; Wiens et al.

The elastic cross sections due to all four symmetries are shown in Figure 3a for SE and the 12-states CC models. These results are without the inclusion of long-range effects. The dominant contribution to cross sections comes from ${ }^{2} A_{1}$ symmetry. The ${ }^{2} B_{1}$ and ${ }^{2} B_{2}$ symmetries are degenerate and thus have same cross sections. The scattering symmetry ${ }^{2} A_{2}$ had a minimal contribution. Beyond $7 \mathrm{eV}$, the elastic cross sections for the corresponding symmetries practically merged with each other. The impact of polarisation on scattering parameters due to various models is shown in Figure 3b. The rapid rise of elastic cross sections when the electron energy decreases is due to the dipolar nature of the molecule. The polarisation effects are important only in the lower electron-energy range. After $7 \mathrm{eV}$, the different scattering models show a merging trend in cross sections. The resonance peak in ${ }^{2} A_{1}$ was visible in the SE model but gets shifted to lower energy values in scattering models, which include polarisation effects. Finally, this resonance disappears in the 12-states CC model as the scattering state becomes a bound anionic state.
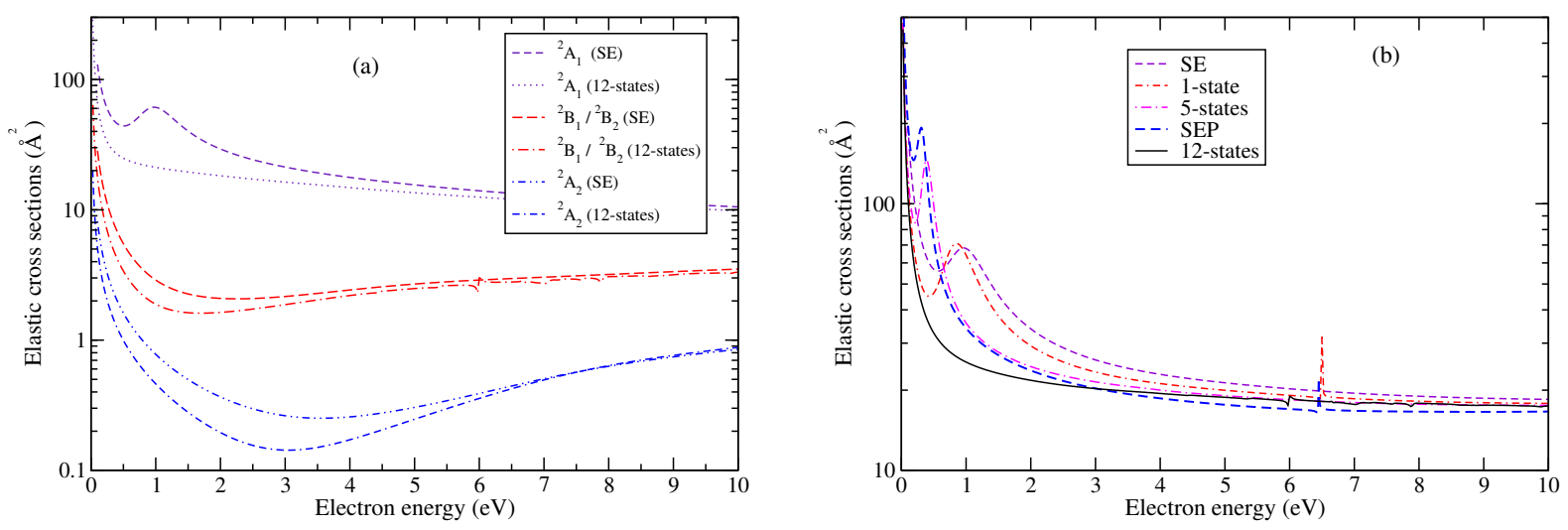

Figure 3. (a) Symmetry wise elastic cross sections in the SE and the 12-states CC model. (b) Elastic cross sections obtained in different scattering models to highlight the effect of polarisation on scattering. These results do not include the long-range effects.

The values of $E^{r e s}$ and $\Gamma^{r e s}$ for the ${ }^{2} A_{1}$ scattering symmetry for all our scattering models are listed in Table 3. It is observed from Table 3 and Figure $3 \mathrm{~b}$ that the shape resonance energy in ${ }^{2} A_{1}$ scattering symmetry shifts to lower energy values as we move from SE to a better scattering model. Additionally, $\Gamma^{\text {res }}$ decreases. 
Table 3. $E^{r e s}$ and $\Gamma^{r e s}$ values in different scattering models for ${ }^{2} A_{1}$ scattering symmetry.

\begin{tabular}{ccc}
\hline $\begin{array}{c}\text { Scattering } \\
\text { Model }\end{array}$ & $\begin{array}{c}\boldsymbol{E}^{\text {res }} \\
(\mathbf{e V})\end{array}$ & $\begin{array}{l}\boldsymbol{\Gamma}^{\text {res }} \\
\mathbf{( e V )}\end{array}$ \\
\hline SE & 0.94 & 0.75 \\
SEP & 0.31 & 0.18 \\
1-state & 0.79 & 0.62 \\
5-states & 0.40 & 0.24 \\
12-states & Bound & - \\
\hline
\end{tabular}

The Born-corrected elastic cross sections in the 12-states CC model are displayed in Figure 4. The correction was obtained via the Born Top-Up Program [22] using the experimental value of the dipole moment [41] and is also plotted in the same figure. The results without Born corrections are helpful in drawing comparisons with the experimental results, which lack forward angle correction [41].

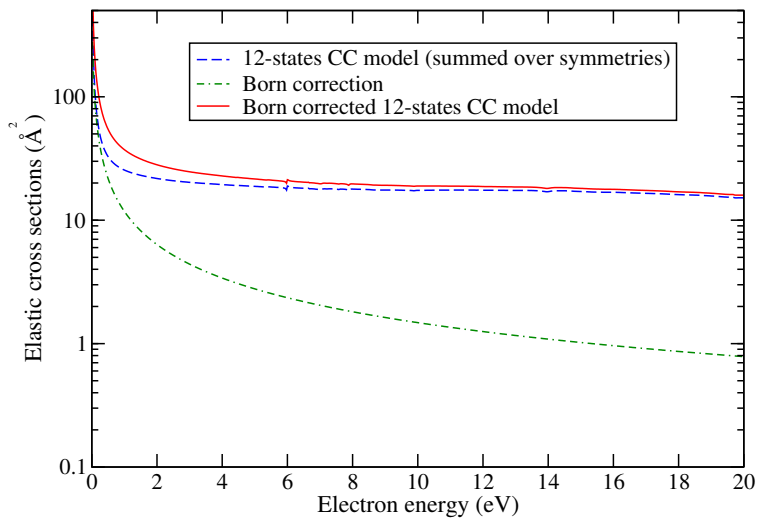

Figure 4. Elastic cross sections in the 12-states CC model. Born-corrected elastic cross sections, line curve; Born correction, dashed dotted curve; elastic cross sections without Born correction, dashed curve.

Wiens et al. [26] in their study on electron attachment from the interhalogen compounds have shown that the resonant state becomes an anionic state as the bond length is increased gradually from lower bond lengths up to the equilibrium bond length. This work was done using the $R$-matrix-based QUANTEMOL code [42] by employing the cc-pVTZ basis set. The active space is the same in both the works. The present study supports the claim of Wiens et al. [26].

The electron-impact excitation cross sections $\left(Q_{e e}\right)$ for spin-forbidden transition $X \rightarrow{ }^{3} \Pi$ and dipole-allowed $X \rightarrow{ }^{1} \Pi$ transition are shown in Figure 5a,b, respectively. The transition moment from $X \rightarrow{ }^{1} \Pi$ was 0.1778 au. These cross sections increase from respective thresholds. The rise in $Q_{e e}$ for the $X \rightarrow{ }^{3} \Pi$ transition was almost linear from the threshold up to $5 \mathrm{eV}$ and before becoming constant. The excitation cross sections for the $X \rightarrow{ }^{1} \Pi$ transition increased almost linearly from the threshold up to $5 \mathrm{eV}$ and then decreased slowly up to $10 \mathrm{eV}$. The excitation cross sections for the dipole transition were also obtained in the Born approximation [43] by replacing the dipole moment with the transition moment of the spin-allowed transition and the rotational excitation energy by the excitation threshold. There is a significant difference between the excitation cross sections obtained in the 12-states CC model and the Born approximation indicating that the effect of correlation is important. Further, the $\mathrm{Q}_{e e}$ obtained for the 5- and 12-states CC models were identical because of the absence of close-coupling channels for the transitions considered in this work. Hence, the results are presented for the 12-states CC model, which was our best model. 

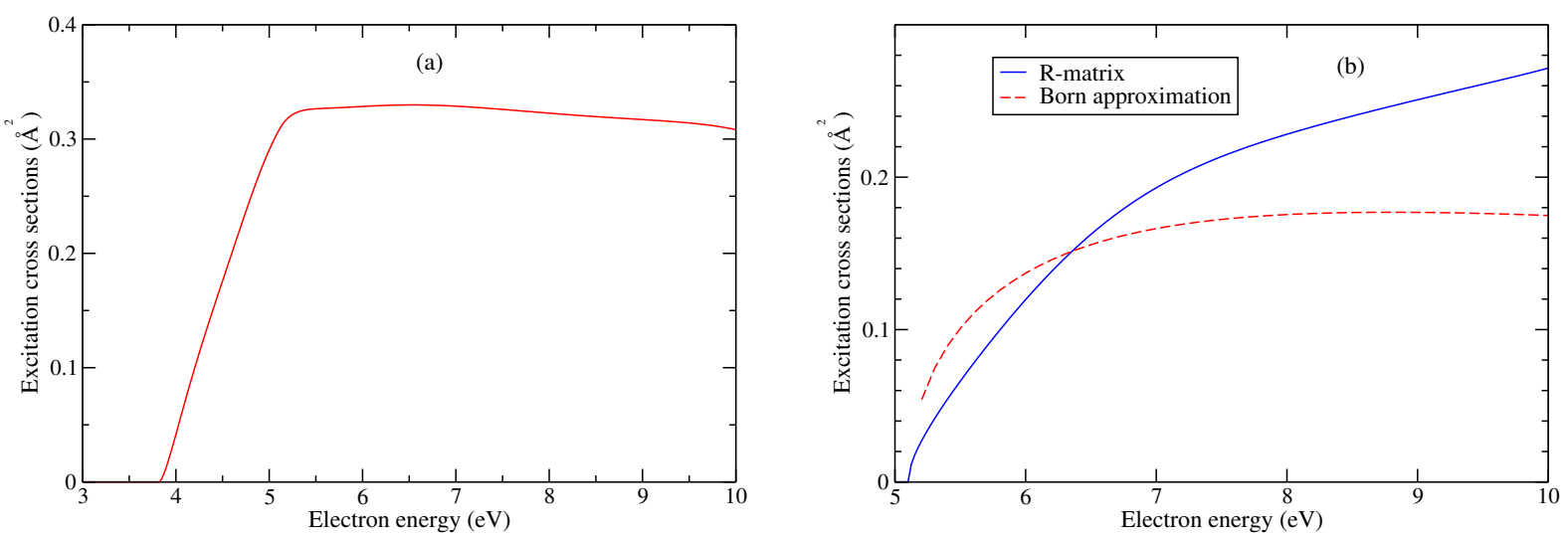

Figure 5. Electronic-excitation cross sections in 12-states CC model (a) ground to spin-forbidden transition transition ${ }^{1} X \rightarrow{ }^{3} \Pi$ and $(\mathbf{b})$ ground to dipole-allowed transition transition ${ }^{1} X \rightarrow{ }^{1} \Pi$, line curve; Born approximation, dashed curve.

The rate coefficient has significance in the fields like plasma and astronomy and can be obtained using the cross-section data. Assuming that the given process obeys a Maxwellian distribution of energy, the rate coefficient $[44,45]$ is obtained for an elastic process in the 12-states CC model. The results are shown in Figure 6. The rates increase from $100 \mathrm{~K}$ up to $3000 \mathrm{~K}$ beyond which these decrease slowly up to $10,000 \mathrm{~K}$.

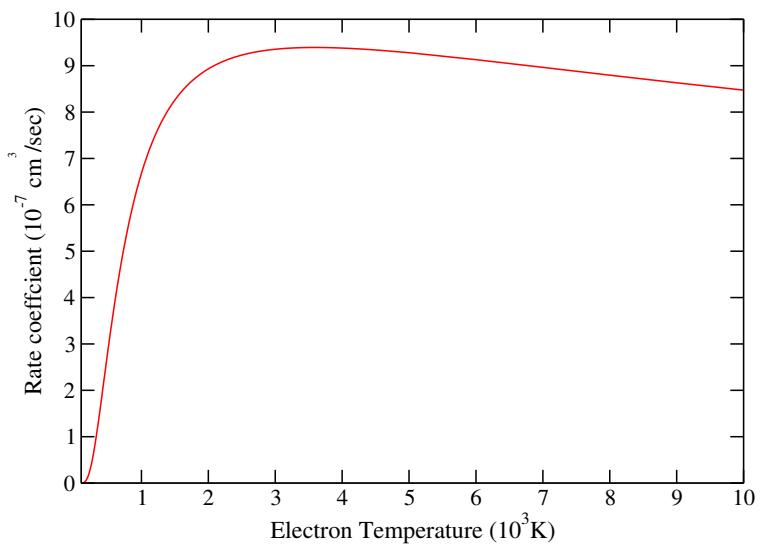

Figure 6. Elastic rate coefficients in the 12-states CC model.

The converged DCS for polar molecules in the lab frame are given by [34]:

$$
\frac{d \sigma}{d \Omega}\left(J \rightarrow J^{\prime}\right)=\frac{d \sigma^{B}}{d \Omega}+\sum_{L}\left(A_{L}-A_{L}^{B}\right) P_{L}(\cos \theta)
$$

where, $P_{L}$ is a Legendre polynomial and $A_{L}$ are the expansion coefficients [46]. The superscript $B$ denotes the quantity obtained in the Born approximation. The first term of Equation (3) represents the rotationally excited target in the laboratory frame computed in the Born approximation. The second term represents the cross sections obtained in the CC model. The last term is an electron-dipole-dominated interaction term and represents the contributions from the lowest five partial waves (from $l=0$ to 4 ). By summing over all the rotational transitions involving $J^{\prime}$, the rotationally unresolved DCS were obtained.

$$
\frac{d \sigma^{\mathcal{S}}}{d \Omega}=\sum_{J^{\prime}} \frac{d \sigma}{d \Omega}\left(J \rightarrow J^{\prime}\right)
$$

Figure 7a shows the DCS obtained using POLYDCS [47] for the energies 1, 3, 6, 8, and $9 \mathrm{eV}$. The requirement of using POLYDCS is to use only the open channels. In the 12-states CC model, both the open and closed channels are generated. All channels up to the first 
excitation threshold of $3.84 \mathrm{eV}$ are open. The DCS calculations were therefore performed at truncated K-matrices in the 12-states CC model so that the validity of POLYDCS was not violated. The DCS showed a pronounced minima around $110-120^{\circ}$ at 6,8 , and $9 \mathrm{eV}$. The sharp increase in DCS for angles below $5^{\circ}$ is a reflection of the polar nature of the target.
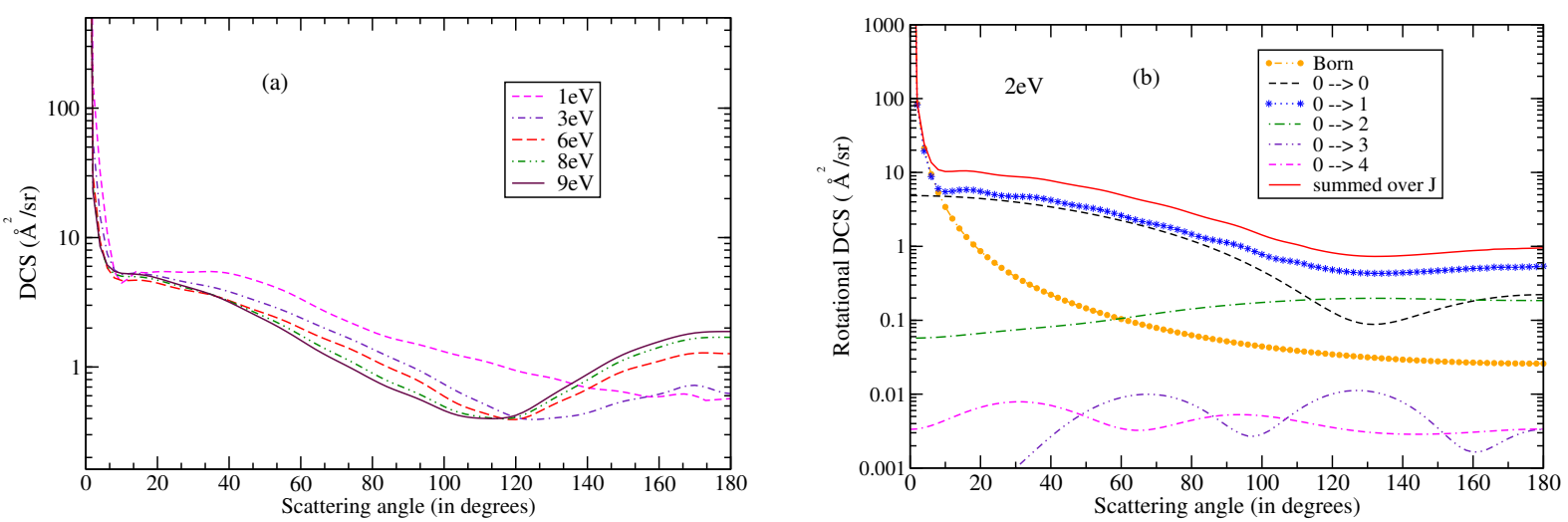

Figure 7. (a) Rotationally summed DCS at different incident energies obtained using 12-states CC model. $1 \mathrm{eV}$; dashed curve, $3 \mathrm{eV}$; dotted dashed curve, big dashed curve; $6 \mathrm{eV}$ and $8 \mathrm{eV}$; double dotted dashed curve, $9 \mathrm{eV}$; line curve (b) Rotationally resolved and summed DCS at $2 \mathrm{eV}: 0 \rightarrow 0$ transition, dashed curve; $0 \rightarrow 1$ transition, dotted curve with star; $0 \rightarrow 2$ transition, dotted dashed curve; $0 \rightarrow 3$ transition, double dotted dashed curve; $0 \rightarrow 4$ transition, dashed dotted curve; circles, summed ( $0 \rightarrow$ $J$ ); circle with dotted line curve, Born approximation; dots.

In Figure 7b, we plotted rotationally resolved DCS at $2 \mathrm{eV}$ and reported the convergence with respect to the rotational angular-momentum $J$ values. The transition $0 \rightarrow 1$ was predominant at scattering angles below $15^{\circ}$. This is expected as this transition is driven by a weak dipole moment of the target molecule. The elastic transition $0 \rightarrow 0$ decreased from the threshold value of $4.8 \AA^{2}$ to a minimum value of $0.02 \AA^{2}$ around $125^{\circ}$ beyond which it increased to $0.36 \AA^{2}$ in the backward angle. This minimum is due to rainbow scattering [48]. The quadrupole transition $0 \rightarrow 2$ is nearly constant in the entire angular range. This behaviour is typical of quadrupole transitions. It is seen that the DCS has converged as the contributions from other $J$ values beyond 2 are minimal. The DCS are also plotted in the Born approximation.

The DCS are used to obtain momentum-transfer cross sections $\left(Q^{m}\right)$ and higherorder collision moments [44]. When the DCS are integrated over the scattering angle, the weighted give momentum-transfer cross sections $Q^{m}$ :

$$
Q^{m}=2 \pi \int_{0}^{\pi}(1-\cos \theta) \sin \theta \frac{d \sigma^{\mathcal{s}}}{d \theta} d \theta
$$

The $Q^{m}$ is required to obtain molecular bremsstrahlung cross sections, diffusion parameters, and collision frequencies $[45,49]$. In Figure 8, we show $Q^{m}$ for electron impact on $\mathrm{ClF}$ molecule in the 1-state and 12-states CC models. The threshold value of $Q^{m}$ had a finite value of around $70 \AA^{2}$ and $50 \AA^{2}$, respectively, in the two CC models. The impact of correlation-polarisation was visible only up to $3 \mathrm{eV}$. There was no singularity problem in $Q^{m}$ due to the weighting factor $(1-\cos \theta)$. With the increasing projectile energy, the $Q^{m}$ decreased up to $3 \mathrm{eV}$ beyond which it was a nearly constant value of $11 \AA^{2}$. This behaviour is correlated with the DCS trends. 


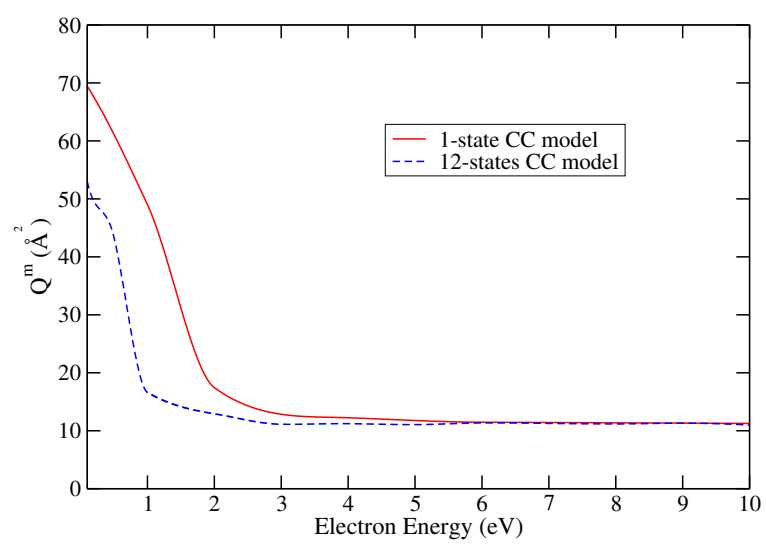

Figure 8. Born-corrected momentum-transfer cross sections.

The collision frequency is an important quantity to determine the transport properties of the electrons in ionospheric and atmospheric physics [50] and is used to obtain collision parameters like the diffusion coefficient, the electron mean-free-path, and mobility [51]. The collision frequencies like $\langle v\rangle$ and $\bar{v}^{-1}$ are plotted in Figure 9 assuming the Maxwell-Boltzmann distribution for the electrons for the temperature ranging from $100 \mathrm{~K}$ to $10,000 \mathrm{~K}[44,45,49]$.

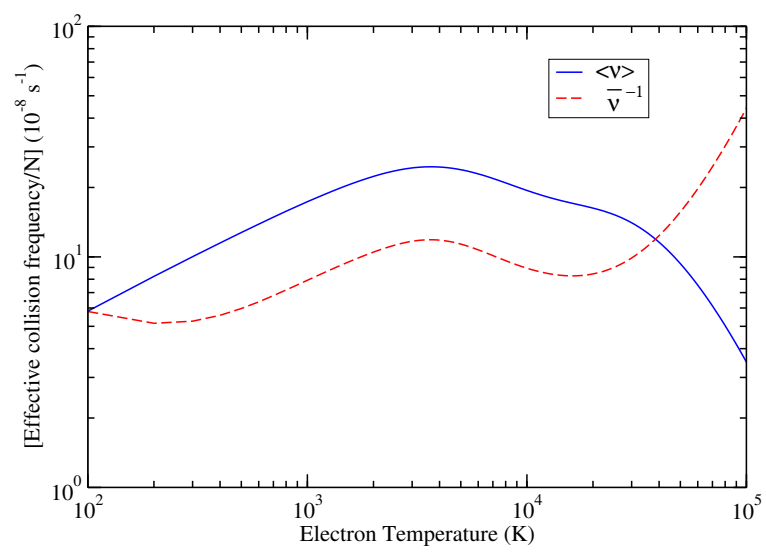

Figure 9. Effective collision frequencies: line curve, $\langle v\rangle$; dashed curve, $\bar{v}^{-1}$.

Denoting $\sigma_{i}$ as the ionization cross sections for the $i$ th-occupied molecular orbital, then the total ionization cross sections $Q^{B E B}$ for singly charged ions in the BEB model [52] are obtained by summing the ionization cross sections over all the occupied molecular orbitals.

$$
Q^{B E B}=\sum_{i} \sigma_{i}(t)
$$

where,

$$
\sigma_{i}(t)=\frac{S}{u+t+1}\left[\frac{1}{2}\left(1-\frac{1}{t^{2}}\right) \ln t+\left[\left(1-\frac{1}{t}\right)-\frac{\ln t}{t+1}\right]\right]
$$

here, $t=T / B, u=U / B$, and $S=4 \pi a_{0}^{2} N(R / B)^{2}$. T, B, and $U$ denotes the energy of the incident electron, the binding energy of the orbital, and the orbital kinetic energy, respectively; $N$ is the electron occupation number, and $R$ is the Rydberg energy. The BEB input parameters like the $B, U$, and $N$ for occupied molecular orbitals are listed in Table 4 . The BEB ionization cross sections are plotted in Figure 10. The cross sections were computed by considering the experimental value of ionization threshold. The cross sections showed a peak value of $3.41 \AA^{2}$ at $110 \mathrm{eV}$ and then showed $\ln E / E$ behaviour at higher projectile energies. We also plotted the cross sections by considering only the valence $3 \pi$ orbitals and by freezing 16 electrons. The $3 \pi$ orbitals contribute a maximum to ionization cross 
sections. It also showed orbitals $5 \sigma$ and $6 \sigma$ orbitals also contribute significantly to TICS. The contribution of core orbitals was negligibly small.

Table 4. Molecular orbital binding energies, average kinetic energies, and occupation number of occupied molecular orbitals obtained at equilibrium geometry at the HF level using a 6-311G* basis set. The orbitals given in the parentheses correspond to $C_{2 v}$ point group.

\begin{tabular}{cccc}
\hline Molecular Orbital & $|\boldsymbol{B}| \mathbf{( e V )}$ & $\mathbf{U}(\mathbf{e V})$ & $\mathbf{N}$ \\
\hline $1 \sigma\left(1 a_{1}\right)$ & 2855.47 & 3731.21 & 2 \\
\hline $2 \sigma\left(2 a_{1}\right)$ & 717.01 & 1013.66 & 2 \\
\hline $3 \sigma\left(3 a_{1}\right)$ & 289.97 & 593.89 & 2 \\
\hline $1 \pi\left(1 b_{1}, 1 b_{2}\right)$ & 221.94 & 562.46 & $4(2,2)$ \\
\hline $4 \sigma(4 a 1)$ & 221.10 & 560.52 & 2 \\
\hline $5 \sigma\left(5 a_{1}\right)$ & 44.69 & 101.877 & 2 \\
\hline $6 \sigma\left(6 a_{1}\right)$ & 30.14 & 91.28 & 2 \\
\hline $2 \pi\left(2 b_{1}, 2 b_{2}\right)$ & 19.76 & 81.61 & $4(2,2)$ \\
\hline $7 \sigma\left(7 a_{1}\right)$ & 19.00 & 79.52 & 2 \\
\hline $3 \pi\left(3 b_{1}, 3 b_{2}\right)$ & 13.44 & 72.43 & $4(2,2)$ \\
\hline
\end{tabular}

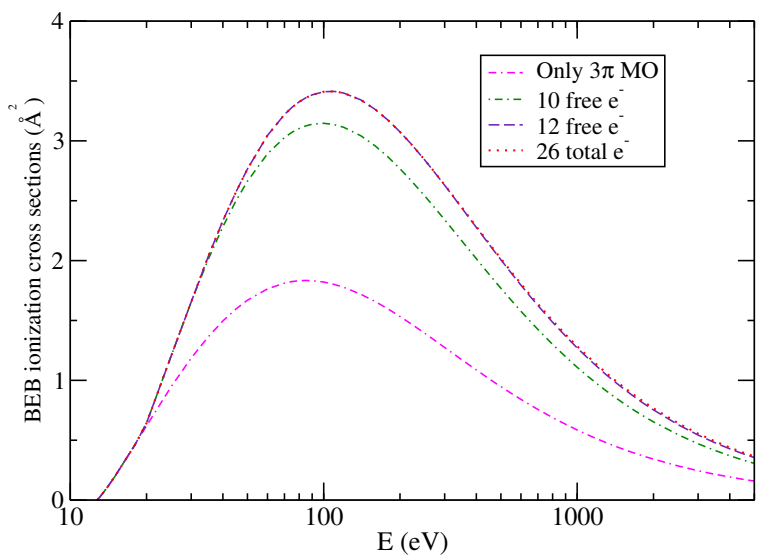

Figure 10. BEB ionization cross section using all electron models by considering different free numbers of electrons: dashed dotted curve, only $3 \pi$ molecular orbitals $(\mathrm{MO})$; dotted dashed curve, 10 free electrons; double dashed curve, 12 free electrons; dotted curve, from total 26 electrons.

\section{Discussion}

The cross sections for electron interactions with $\mathrm{ClF}$ were reported using the $R$-matrix method. These cross sections were computed in different models like SE, SEP, and CC models to understand the effect of correlation and polarisation on scattering and resonance formation. Only one shape resonance was detected in ${ }^{2} A_{1}$ scattering symmetry in SE, SEP, 1-state, and 6-states models. The resonance and width positions decrease to lower values as polarisation effects increase with the inclusion of more target states. This shape resonance becomes bound in the 12-states CC model due to the inclusion of polarisation effects. The 12-states CC model is our best model as it includes adequate polarisation effects to obtain the anionic bound state ${ }^{2} A_{1}$ of $\mathrm{ClF}$. This model also provides electronic excitation cross sections for dipole-allowed and -forbidden transitions. The vertical electron infinity of the bound state is in excellent agreement with the value obtained from sophisticated correlation effects. The vertical excitation energies obtained in the 12-states CC model showed good agreement with the MRD calculations. The Born-corrected DCS were reported from $1 \mathrm{eV}$ to $10 \mathrm{eV}$. In addition to the vertical ionization potential and electron affinity, other physical and chemical properties like the dipole moment and quadrupole moments were also reported. 
The vibrational cross sections for the infra-red transition were also reported, which have orders of magnitude smaller than the rotational cross sections. The cross-section data obtained in CC models were used to report collision frequencies and rate coefficients. These quantities are of interest to the plasma community.

Author Contributions: Conceptualization, supervision, and methodology, K.L.B.; calculations, K.L.B. and A.B.; writing the original draft, M.B., A.B. and K.L.B.; review and editing, A.B. and K.L.B. All authors have read and agreed to the published version of the manuscript.

Funding: This research received no external funding.

Institutional Review Board Statement: Not applicable.

Informed Consent Statement: Not applicable.

Data Availability Statement: Can be obtained from the authors on request.

Conflicts of Interest: The authors declare no conflict of interest.

$\begin{array}{ll}\text { Abbreviations } \\ \text { BEB } & \text { Binary-encounter-Bethe } \\ \text { HF } & \text { Hartree-Fock } \\ \text { SE } & \text { Static-Exchange } \\ \text { SEP } & \text { Static-Exchange-polarisation } \\ \text { CI } & \text { Configuration Interaction } \\ \text { CC } & \text { Close Coupling } \\ \text { CASCI } & \text { Complete Active Space Configuration Interaction } \\ \text { CSF } & \text { Configuration State Functions } \\ \text { DCS } & \text { Differential Cross Sections } \\ \text { TICS } & \text { Total Ionization Cross Sections } \\ \text { MO } & \text { Molecular Orbitals } \\ \text { eV } & \text { Electron Volt } \\ \text { au } & \text { Atomic Unit } \\ \text { SCF } & \text { Self-Consistent Field }\end{array}$

\section{References}

1. Ewig, C.S.; Sur, A.; Banna, M.S. A theoretical study of core and valence ionization in ClF. J. Chem. Phys. 1981, 75, 5002. [CrossRef]

2. Law, C.K.; Chien, S.H.; Li, W.K. Thermochemistry of Chlorine Fluorides $\mathrm{ClF}_{n}, \mathrm{n}=1-7$. and Their Singly Charged Cations and Anions: A Gaussian-3 and Gaussian-3X Study. J. Phys. Chem. A 2002, 106, 11271. [CrossRef]

3. Eden, J.G.; Dlabal, M.L.; Hutchison, S.B. The interhalogens IF and ICl as Visible Oscillators or Amplifiers. IEEE J. Quantum Electron. 1981, 17, 1085. [CrossRef]

4. Diegelmann, M.; Hohla, K.; Rebentrost, F.; Kompa, K.L. Diatomic interhalogen laser molecules: Fluorescence spectroscopy and reaction kinetics. J. Chem. Phys. 1982, 76, 1233. [CrossRef]

5. Diegelmann, M.; Proch, D.; Zhensheng, Z. Discharge pumped ClF laser at $285 \mathrm{~nm}$. Appl. Phys. B 1986, 40, 49. [CrossRef]

6. Naulin, C.; Bougon, R. Photochemistry of the chlorine monofluoride, ClF. Thermal and photochemical reactions with sulfur tetrafluoride $\mathrm{SF}_{4}$. J. Chem. Phys. 1980, 72, 2155. [CrossRef]

7. Ibbotson, D.E.; Mucha, J.A.; Flamm, D.L. Plasmaless dry etching of silicon with fluorine-containing compounds. J. Appl. Phys. 1984, 56, 2939. [CrossRef]

8. Takahashi, Y.; Kato, K.; Habuka, H. Development of SiC Etching by Chlorine Fluoride Gas. Mater. Sci. Forum 2020, $1004,731$. [CrossRef]

9. Bargainer, M.; Dietrich, P.; Schwentner, N. Spectroscopy and photodissociation of ClF in rare gas solids. J. Chem. Phys. 2001, $115,149$.

10. Naulin, C.; Lambard, J.; Bougon, R. Vibrational spectra of the chlorine monofluoride ClF in cryogenic solutions. J. Chem. Phys. 1982, 76, 3371. [CrossRef]

11. Krogh, O.D.; Pimentel, G.C. Chemical Lasers from the Reactions of $\mathrm{ClF}$ and $\mathrm{ClF}_{3}$ with $\mathrm{H}_{2}$ and $\mathrm{CH}_{4}$ : A Possible Chain-Branching Chemical Laser. J. Chem. Phys. 1972, 56, 969. [CrossRef]

12. Krogh, O.D.; Pimentel, G.C. $\mathrm{ClF}_{x}-\mathrm{H}_{2}$ chemical lasers $(\mathrm{x}=1,3,5)$ : Vibration-rotation emission by HF from states with high rotational excitation. J. Chem. Phys. 1977, 67, 2993. [CrossRef]

13. Cuellar, E.; Pimentel, G.C. Rotational laser emission by HF in the ClF-H $H_{2}$ chemical laser. J. Chem. Phys. 1979, 71, 1385. [CrossRef]

14. Pimentel, G.C.; Krogh, O.D. Vibrational emission by $\mathrm{HCl}$ from the $\mathrm{ClF}_{2} \mathrm{H}_{2}$ chemical laser. J. Chem. Phys. 1980, 73, 120. [CrossRef] 
15. Alekseyev, A.B.; Liebermann, H.P.; Buenker, R.J.; Kokh, D.B. Relativistic configuration interaction study of the ClF molecule and its emission spectra from $\mathrm{O}^{+}$ion-pair states. J. Chem. Phys. 2000, 112, 2274. [CrossRef]

16. Li, S.; Chen, S.J.; Zhu, D.S.; Wei, J.J. Structure and Potential Energy Function of $\mathrm{ClF}^{-}$Molecular Ion. Acta Phys.-Chim. Sin. 2013, $29,737$.

17. Horny, L.; Sattelmeyer, K.W.; Schaefer, H.L. Elusive electron affinity of ClF. J. Chem. Phys. 2003, 119, 11615. [CrossRef]

18. Nguyen, M.T.; Ha, T.K. Abinttio calculation of the ionization potentials and hyperfine splitting constants of the radical anions $\mathrm{FCl}^{\mathrm{XXX}}$ and $\mathrm{Cl}_{2}^{\mathrm{XXX}}$. Chem. Phys. Lett. 1987, 136, 413. [CrossRef]

19. Van Huis, T.J.; Galbraith, J.M.; Schaefer, H.F. The monochlorine fluorides $\left(\mathrm{ClF}_{n}\right)$ and their anions $\left(\mathrm{ClF}_{n}^{-}\right) \mathrm{n}=1-7: \mathrm{Structures}$ and energetics Mol. Phys. 1996, 89, 607. [CrossRef]

20. McDermid, I.S. Potential-energy curves, Franck-Condon factors and laser excitation spectrum for the $\mathrm{B}^{3} \Pi\left(\mathrm{O}^{+}\right)-\mathrm{X}^{1} \Sigma^{+}$system of chlorine monofluoride. J. Chem. Soc. Faraday Trans. II 1981, 77, 519. [CrossRef]

21. de Jong, W.A.; Styszynski, J.; Visscher, L.; Nieuwpoort, W.C. Relativistic and correlation effects on molecular properties: The interhalogens $\mathrm{ClF}, \mathrm{BrF}, \mathrm{BrCl}, \mathrm{IF}, \mathrm{ICl}$, and IBr. J. Chem. Phys. 1998, 108, 5177. [CrossRef]

22. Kaur, S.; Baluja, K.L.; Tennyson, J. Electron-impact study of NeF using the R-matrix method. Phys. Rev. A 2008, 77, 032718. [CrossRef]

23. Wang, F.; Liu, J.B.; Sinibaldi, J.; Brophy, C.; Kuthi, A.; Jiang, C.; Ronney, P.; Gundersen, M.A. Transient plasma ignition of quiescent and flowing air/fuel mixtures. IEEE Trans. Plasma Sci. 2005, 33, 844. [CrossRef]

24. Nguyen-Kuok, S. Theory of Low-Temperature Plasma Physics; Springer International Publishing: Cham, Switzerland, 2017

25. Chu, P.K.; Lu, X.P. (Eds.) Low Temperature Plasma Technology: Methods and Applications; CRC Press: Boca Raton, FL, USA, 2014.

26. Wiens, J.P.; Sawyer, J.C.; Miller, T.M.; Shuman, N.S.; Viggiano, A.A.; Khamesian, M.; Kokoouline, V.; Fabrikant, I.I. Electron attachment to the interhalogen compounds ClF, ICl, and IBr. Phys. Rev. A 2016, 93, 032706. [CrossRef]

27. Tennyson, J. Electron-molecule collision calculations using the R-matrix method. Phys. Rep. 2010, 491, 29. [CrossRef]

28. Burke, P.G. R-Matrix Theory of Atomic Collisions; Springer: Berlin/Heidelberg, Germany, 2011.

29. Baluja, K.L.; Burke, P.G.; Morgan, L.A. R-matrix propagation program for solving coupled second-order differential equations. Comput. Phys. Commun. 1982, 27, 299. [CrossRef]

30. Masin, Z.; Benda, J.; Harvey, A.G.; Gorfinkiel, J.D.; Tennyson, J. UKRmol+: A suite for modelling electronic processes in molecules interacting with electrons, positrons and photons using the R-matrix method. Comp. Phys. Commun. 2020, 249, 107092. [CrossRef]

31. Masin, Z.; Benda, J.; Harvey, A.G.; Al-Refaie, A.; Gorfinkiel, J.D.; Tennyson, J. UKRmol+: UKRMol-in (Version 3.0); Zenodo: Genève, Switzerland, 2019. [CrossRef]

32. Johnson, R.D., III (Ed.) Computational Chemistry Comparison and Benchmark Database, Number 101, Release 21, August 2020. Available online: http:/ / cccbdb.nist.gov (accessed on 21 December 2021).

33. Faure, A.; Gorfinkiel, J.D.; Morgan, L.A.; Tennyson, J. GTOBAS: Fitting continuum functions with Gaussian-type orbitals. Comput Phys. Commun. 2002, 144, 224. [CrossRef]

34. Fabrikant, I.I. Long-range effects in electron scattering by polar molecules. J. Phys. B At. Mol. Opt. Phys. 2016, $49,222005$. [CrossRef]

35. Bassi, M.; Bharadvaja, A.; Baluja, K.L. A study of electron scattering from $1-1 \mathrm{C}_{2} \mathrm{H}_{2} \mathrm{~F}_{2}$ from $0.1 \mathrm{eV}$ to 5 keV. Eur. Phys. J. D 2020 , 74, 232. [CrossRef]

36. Ewing, J.J.; Tigelaar, H.L.; Flygare, W.H. Molecular Zeeman Effect, Magnetic Properties, and Electric Quadrupole Moments in ClF, BrF, ClCN, BrCN, and ICN J. Chem. Phys. 1972, 56, 1957. [CrossRef]

37. Vassilakis, A.A.; Kalemos, A.; Mavridis, A. Accurate first principles calculations on chlorine fluoride $\mathrm{ClF}$ and its ions $\mathrm{ClF}^{ \pm}$. Theor Chem. Acc. 2014, 133, 1436. [CrossRef]

38. Quintero-Monsebaiz, R.; Perea-Ramírez, L.I.; Piris, M.; Vela, A. Spectroscopic properties of open shell diatomic molecules using Piris natural orbital functionals. Phys. Chem. Chem. Phys. 2021, 23, 2953. [CrossRef] [PubMed]

39. Darvesh, K.V.; Boyd, R.J.; Peyerimhoff, S.D. Electronically excited states of chlorine monofluoride: A multi-reference configuration interaction study. Chem. Phys. 1988, 121, 361. [CrossRef]

40. Tennyson, J.; Noble, C.J. RESON-A program for the detection and fitting of Breit-Wigner resonances. Comput. Phys. Commun. 1984, 33, 421. [CrossRef]

41. Sahgal, V.; Bharadvaja, A.; Baluja, K.L. Positron-induced scattering of acetone from $0.1 \mathrm{eV}$ to 5 keV. J. Phys. B At. Mol. Opt. Phys. 2021, 54, 075202. [CrossRef]

42. Cooper, B.; Tudorovskaya, M.; Mohr, S.; O’Hare, A.; Hanicinec, M.; Dzarasova, A.; Gorfinkiel, J.D.; Benda, J.; Mašín, Z.; Al-Refaie, A.F.; et al. Quantemol Electron Collision: An expert system for performing UKRmol+ electron molecule collision calculations. Atoms 2019, 7, 97. [CrossRef]

43. Itikawa, Y. Molecular Processes in Plasmas; Springer: Berlin/Heidelberg, Germany, 2007.

44. Bharadvaja, A.; Kaur, S.; Baluja, K.L. Electron-impact cross sections of $\mathrm{SiH}_{2}$ using the R-matrix method at low energy. Phys. Rev. A 2015, 91, 032701. [CrossRef]

45. Bharadvaja, A.; Kaur, S.; Baluja, K.L. Low-energy electron impact cross-sections and rate constants of $\mathrm{NH}_{2}$. Pramana-J. Phys. 2017, 89, 30. [CrossRef]

46. Gianturco, F.A.; Jain, A. The theory of electron scattering from polyatomic molecules. Phys. Rep. 1986, 143, 347. [CrossRef] 
47. Sanna, N.; Gianturco, F.A. Differential cross sections for electron/positron scattering from polyatomic molecules. Comput. Phys. Commun. 1998, 114, 142. [CrossRef]

48. Sarma, G.; Saha, A.K.; Bishwakarma, C.K.; Scheidsbach, R.; Yang, C.H.; Parker, D.; Wiesenfeld, L.; Buck, U.; Mavridis, L.; Marinakis, S. Collision energy dependence of state-to-state differential cross sections for rotationally inelastic scattering of $\mathrm{H}_{2} \mathrm{O}$ by He. Phys. Chem. Chem. Phys. 2017, 19, 4678. [CrossRef]

49. Kaur, S.; Bharadvaja, A.; Baluja, K.L. Electron-impact study of $\mathrm{S}_{3}$ using the R-matrix method. Phys. Rev. A 2011, 27, 062707. [CrossRef]

50. Itikawa, Y. Effective collision frequency of electrons in atmospheric gases. Planet. Space Sci. 1971, 19, 993. [CrossRef]

51. Baille, P.; Chang, J.S.; Claude, A.; Hobson, R.M.; Ogram, G.L.; Yau, A.W. Effective collision frequency of electrons in noble gases. J. Phys B At. Mol. Phys. 1981, 14, 1485. [CrossRef]

52. Kim, Y.-K.; Rudd, M.E. Binary-encounter-dipole model for electron-impact ionization. Phys. Rev. A 1994, 50, 3954. [CrossRef] [PubMed] 